\title{
The Value of Patient-Reported Outcome (PRO) Data in Digital Healthcare: Using the How-R-you App as a PRO Instrument
}

\author{
Sune Dueholm Müller \\ Aarhus University \\ sdm@processinnovation.dk
}

\author{
Morten Mathiasen \\ Business Academy Aarhus \\ mmat@eaaa.dk
}

\author{
Birgitte Mahler \\ Aarhus University Hospital \\ mahler@,clin.au.dk
}

\begin{abstract}
This paper evaluates the value of using a mobile digital technology for the purpose of collecting PatientReported Outcome (PRO) data. We compare the use of a paper-based questionnaire and a mobile application as two different PRO instruments. Based on analysis, we conclude that use of the mobile application allows for a more nuanced picture of patients' health to be established. Implications for diagnosis and medical treatment are discussed along with the need for future research.
\end{abstract}

\section{Introduction}

A recent study by the OECD concluded that health spending has outpaced economic growth in member countries for the past 20 years, and that the rising healthcare costs are unsustainable without reform (http://www.oecd.org/health/healthcarecostsunsustaina bleinadvancedeconomieswithoutreform.htm).

Innovation of healthcare services is needed if the level of quality in healthcare is to be maintained [15]. This innovation is in part driven by emerging digital technologies, e.g. mobile technologies. Concurrently, public sector strategies like the one in Denmark call for patients playing a larger role in taking responsibility for their own health and medical treatment. Thus, the Danish Ministry of Health has defined 8 goals for the provision of national healthcare services [23] in collaboration with Local Government Denmark and Danish Regions. Increased patient involvement is among these goals. Meanwhile, patient-centered healthcare requires active engagement of patients [19]. In executing the strategy, use of digital technology is one of the means by which to increase involvement and patient empowerment. However, to ensure that the technology contributes to value creation, designers and developers of digital technology need to understand and consider the interests and needs of both patients and healthcare professionals. Our current knowledge is, however, limited and needs to be expanded through research.

Technology has the potential to provide both healthcare professionals and patients with information in support of diagnosis and treatment. Doctors need accurate information to provide effective and costefficient healthcare. Patients - especially with chronic diseases - need information in order to help themselves and self-manage their medical conditions. In many instances, both parties have incomplete information about patients' health and how their disease progresses [8]. In the case of patients with chronic diseases who need long-term healthcare, being able to continually monitor the patients and follow any developments is important from the perspective of doctors. Today, doctors rely to a large extent on test results (from periodic blood tests, scans etc.) and dialogue with patients. In connection with check-ups, patients are asked to account for not only their well-being and state of health right now, but also how they have been since their last check-up, e.g. whether they have experienced any pain and discomfort, and whether their disease has impacted their daily lives. The reliability of the dataor information - provided by the patients is low due to their difficulties in recalling the details of everyday life with any accuracy [20].

The question is whether doctors can secure more reliable information about patients' health, and how data can be collected to that end by means of technology both automatically and manually. Data can be collected automatically by for example tracking patients' vital signs and physical movements through sensor technology. In addition, patients can be encouraged to manually report observations of a more subjective nature regarding their health by making it convenient, for example through mobile applications. This leads us to our research question: To what extent can digital PRO instruments be used to establish a more accurate picture of patients' health? We address this question in the context of a research project that investigates the relationships between pain, sleep, and disease activity in an attempt to identify causes of reduced physical activities and pain among children and adolescents 
diagnosed with arthritis. We focus in particular on the value from the perspective of doctors of using a mobile application for collecting Patient-Reported Outcome (PRO) data. To that end, we compare it to a currently used PRO instrument - a paper-based questionnaire. In other words, we evaluate the efficacy of a digital PRO instrument as compared to conventional, paper-based methods of patient data collection. Based on our analysis, we conclude that the app provides more nuanced if not more accurate information, which opens for new healthcare possibilities and challenges.

The paper is structured as follows. First, we account for state-of-the-art knowledge of digital healthcare with a specific focus on mHealth and technology support for PRO data collection. Second, we describe our research approach, including the use of an app that has been developed and tested as part of this study. Third, we present our findings, comparing questionnaire and app data through statistical analyses. Fourth, we discuss the results in relation to extant literature, stressing the contribution to mHealth research and the value of app technology for PRO data collection.

\section{Background literature}

In this section, we account for extant research within mHealth, focusing in particular on digital technologies as instruments for collecting and analyzing PRO data.

According to the World Health Organization there is no single, agreed-upon definition of mHealth. In their global survey of eHealth, mHealth (mHealth is a component of eHealth) is defined as "medical and public health practice supported by mobile devices, such as mobile phones, patient monitoring devices, personal digital assistants (PDAs), and other wireless devices" [28:6]. This is mirrored in the academic literature where mHealth is broadly defined as health service delivery over a mobile or wireless platform: "mHealth is all about delivering health services and information over a mobile platform" [17:671]. mHealth is closely related to digital health. Digital health is the use of digital technologies for the purpose of facilitating patients' participation in the healthcare delivery process "to monitor and improve the wellbeing and health of patients and to empower patients in the management of their health and that of their families" [9:246].

Mobile, digital technologies like smartphones drive development of innovative healthcare solutions due to their unique characteristics, e.g. mobility, accessibility, and the ability to collect and transmit data [11]. The potential for mHealth applications is enormous and includes continuous surveillance of vital signs and physiological well-being, as well as access to virtual care services, drug information, personal electronic medical records, and a wealth of educational resources [14]. This is attracting the interest of practitioners and researchers alike, which is reflected in an increasing use of apps within the healthcare sector [4].

Among healthcare apps are those that are being used for collecting data from patients also known as PatientReported Outcome (PRO) data. According to the U.S. Food and Drug Administration (FDA), "a patient reported outcome (PRO) is a direct response from the patient regarding his/her health condition, without a healthcare provider or caregiver interpretation" (https://www.fda.gov/ForPatients/PatientEngagement/d efault.htm\# PRO ), and PRO instruments like apps are important tools in directly measuring how the patient feels and functions. These apps and mHealth in general are interesting in a health economics perspective, because "a small number of patients, mostly the chronically sick, are disproportionately costly in any health-care system. M-health offers a continuous, longterm means of monitoring them, with the potential to improve the way conditions such as cardiovascular disease, epilepsy, asthma and diabetes are managed" [24]. Chronic non-communicable diseases account for a greater part of "the global disease burden" than that of "communicable diseases, maternal and perinatal conditions, nutritional causes and injuries put together and are expected to increase by 2020" [19:948]. Thus, mHealth has the potential to improve not only the interaction and dialogue between healthcare professional and patients but also the cost and quality of treatment [7].

Doctor-patient consultations can be conducted more efficiently if less time is spent on administration (e.g., accessing and inputting data in various health information systems) and more time in actual physical meetings between patients and doctors [26]. Paradoxically, doctors fear that new technology will increase rather than decrease their administrative burdens, and that their introduction will necessitate time-consuming training and work practice adaptations $[21,26]$. Nevertheless, mHealth introduces a paradigm shift in the sense that mobile technologies stimulate innovation and reconceptualization of medical care. Work processes and routines are being rethought from the perspective of how best to accomplish the main objective of providing unsurpassed medical treatment regardless of how it has been accomplished in the past. The envisioned application areas are numerous. Apps could, for example, be used for data collection, patient monitoring, clinical decision-making, and information management [25].

According to Gallagher et al. (2015), patientcenteredness is key to effective treatment of chronic diseases, which entails involving patients more directly in the treatment process. Regularly registering patient 
data improves the quality of diagnoses and treatment, partly because healthcare professionals are able to adapt plans based on PRO data [6]. PRO data opens up new possibilities, and studies have shown the value of utilizing such data with regard to making early diagnoses and freeing up resources [10]. Turning patients into active participants increases treatment quality from a patient satisfaction perspective [21]. Furthermore, greater involvement contributes positively to patient compliance, which can be defined as "the extent to which a person's behavior-taking medication, following a diet, and/or executing lifestyle changes, corresponds with agreed recommendations from a health care provider" [27:136].

Despite its potential, there are also valid concerns regarding patient involvement. Among other things, the quality of PRO data is limited by patients' abilities (or lack thereof) to remember details about their conditions, which is referred to as recall bias: "A potential for recall bias exists whenever historical self-report information is elicited from respondents" [20:167]. In addition, studies show that motivating patients to use health apps is challenging despite the potential of improving their daily lives. Another challenge is establishing the clinical value of such PRO instruments, i.e. determining whether they improve not only doctors' work practices but more importantly patients' health. Consequently, more research on the value and limitations of digital technology enabled PRO instruments is needed [11].

LoPresti et al. (2015) have examined state-of-the-art research and clinical trials involving mHealth applications worldwide - most of which take place in the setting of chronic diseases-and conclude that "evidence in support of this technology is unclear from the trials included in this study ... Further research should focus on establishing the effectiveness of particular mHealth applications and comparing the effectiveness of these new approaches with that of existing methods" $[13: 17,24]$. Our research contributes to filling that knowledge gap by investigating the extent to which digital technology can be used to establish a more accurate picture of patients' health.

\section{Research design}

In this section, we describe the research background, the case setting, and the instruments that were used for PRO data collection as part of the study. The following subsections will provide detailed descriptions of the app that was developed as part of the research project as well as our approach to data collection and analysis.

This paper reports on the research collaboration between Department of Pediatrics and Adolescent Medicine at Aarhus University Hospital, Business
Academy Aarhus, and Aarhus University. In 2016, a group of doctors at the Department of Pediatrics and Adolescent Medicine initiated a project entitled "Pain, sleep and disturbed circadian rhythm in Juvenile Idiopathic Arthritis" for the purpose of identifying pathological mechanisms behind reduced physical activity and children's pain. The doctors enlisted the help of Business Academy Aarhus to develop the HowR-you app (see below) to empower patients to keep track of their daily symptoms and share the information with the doctors. The app is envisioned as a supplement to and potentially a substitute for more traditional means of collecting data about patients' health.

The Department of Pediatrics and Adolescent Medicine at Aarhus University Hospital is responsible for both surgical and medical healthcare services to children and adolescents in the ages from 0 to 18 years, including pediatric rheumatology. Diagnosing and treating children and adolescents with arthritis is a multidisciplinary teamwork involving doctors, nurses, psychologists, and physiotherapists - all trained and experienced in pediatric rheumatology. The main goal is to detect and aggressively treat arthritis in order to preserve normal joint function and growth.

Despite new medical treatment modalities, this group of patients experience reduced quality of life with pain, decreased physical activity, and sleep disturbances as major contributors to morbidity $[12,18]$. Patients receive routine check-ups in an outpatient clinic between 2 and 6 times a year depending on individual needs. In case of acute arthritis flare, an extra check-up is scheduled and the patient is closely monitored until the disease is under control. Such flares can be detected in patients as painful or swollen joints, but in some cases the symptoms are not manifest. However, a decrease in physical activity and general well-being can be indicative of an arthritis flare. Flares are evaluated by physical examination of all joints, eyes, skin, and mucosa. Biochemistry and imaging can assist the identification of disease activity. Early detection of a flare as well as monitoring disease related symptoms such as chronic pain, sleep disturbances, and decreased physical activity is important in the care of arthritis patients. Doctors rely on PRO data in diagnosing and monitoring the disease. It can, however, be difficult for children and adolescents to recall specific symptoms and accurately report changes in their state of health over time.

With regard to information about patients' health, the doctors depend in part on an internationally recognized questionnaire-Juvenile Arthritis Multidimensional Assessment Report (JAMAR) [3]. JAMAR is a parent/patient-reported outcome measure that is used in assessing patients' physical and mental well-being, including how pain is experienced and influences their 
mood and behavior. It also contains disease and medication specific questions related to arthritis treatment. The questionnaire is intended to be completed prior to a scheduled meeting with the doctor. The answers rely on recalled experiences of disease symptoms during either the last week or last month.

The app was introduced to counter recall bias and to give the children and their parents a tool for continually monitoring their overall state of health in contrast to only assessing it at fixed points in time, i.e. before a check-up. The app serves as a means of developing a multidimensional understanding of pain and the effects of pain on everyday life for children with arthritis. To that end, we designed the questions on the basis of recommendations from the International Association for the Study of Pain, and included the following "core outcome domains and measures that should be considered in clinical trials of treatments for acute and chronic pain in children and adolescents" [16:771]: pain intensity, physical functioning, emotional functioning, and role functioning. In order to automatically monitor physical activity and sleep (physical functioning) we included the GENEActiv device (see below).

\subsection{The How-R-you app}

The idea behind the How-R-you app came from the doctors who were concerned about the low quality of PRO data when paper-based questionnaires are used for data collection purposes. The reliability and validity of such data are questionable because patients report on their health and well-being at fixed points in time rather than on a continuous basis, and they furthermore have to rely on their ability to recall past behaviors and health conditions. The app is envisioned as a substitute for the paper-based questionnaires and as a solution to the data quality problems.

The app is available through the Apple App Store and Google Play. It can also be downloaded here: http://how-r-you.online/en/

The app has been developed in an iterative process involving patients, doctors, UX designers, and programmers. Through requirements elicitation, it became evident that patients want an easy-to-use tool that allows for daily health registrations and which provides an overview of their health history. Additionally, they asked for a free-form text field that allows them to write daily notes in a diary-style form to help them monitor their chronic disease.

How-R-you allows for convenient data collection from patients by means of, e.g., smartphones and tablets. The app contains modularized and configurable health related questions. It also includes a diary and provides an overview of historical data through graphs (see
Figure 2) and tables. The app also integrates with the GENEActiv accelerometer and collects sensor data. GENEActiv is a wrist-worn actigraphy device that is used for temperature, light, and activity monitoring. The sensor data can be analyzed in order to show the patient's level of physical activity and sleep pattern. (https://www.activinsights.com/products/geneactiv/).

All registered data is sent in real time to a central database from which doctors and patients can access the data as needed. The data is password protected and encrypted to ensure that only authorized personnel have access to the data.

The app is designed with user-friendliness in mind. As a substitute for paper-based questionnaires, it is an interactive tool that requires only a few activity steps on the part of users. Both the home screen of the app and the modularized questions are configurable to serve the individual needs of doctors and patients. Due to the feedback from patients and doctors during the design process, the number of questions, their wording, and response formats (e.g., numerical input, multiple choice, and slider scales) are tailorable to individual needs.

In this study, the app is configured to include two modules, "Pain" and "Pain relief", which are accessible through the dashboard on the home screen depending on user settings (see Figure 1).

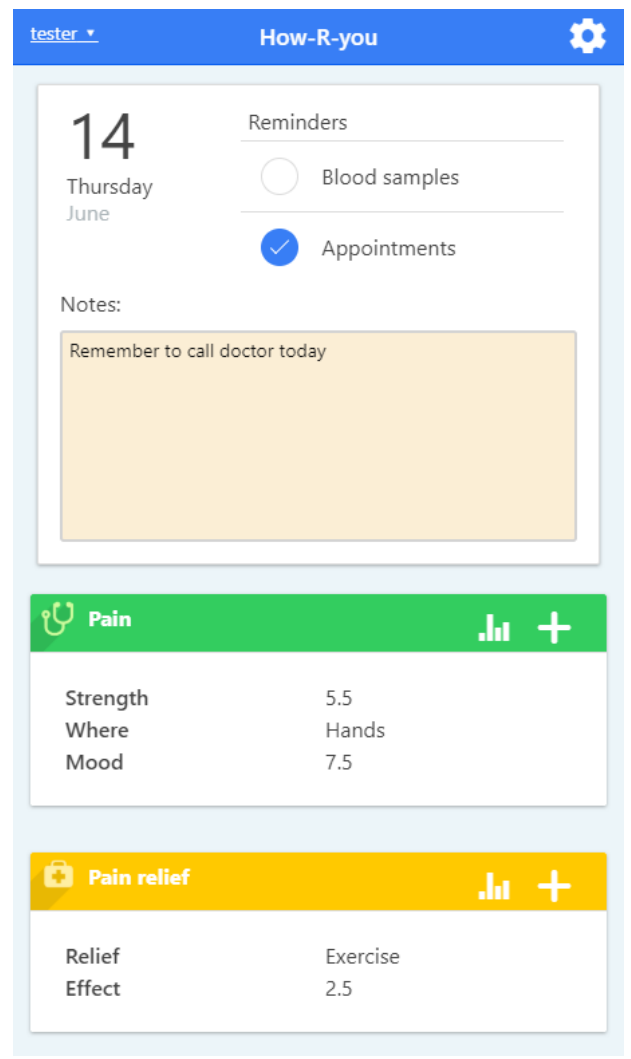

Figure 1. How-R-you home screen 
In addition, it provides an overview of the patient's activity as monitored through the GENEActiv actigraphy device (see Figure 2).

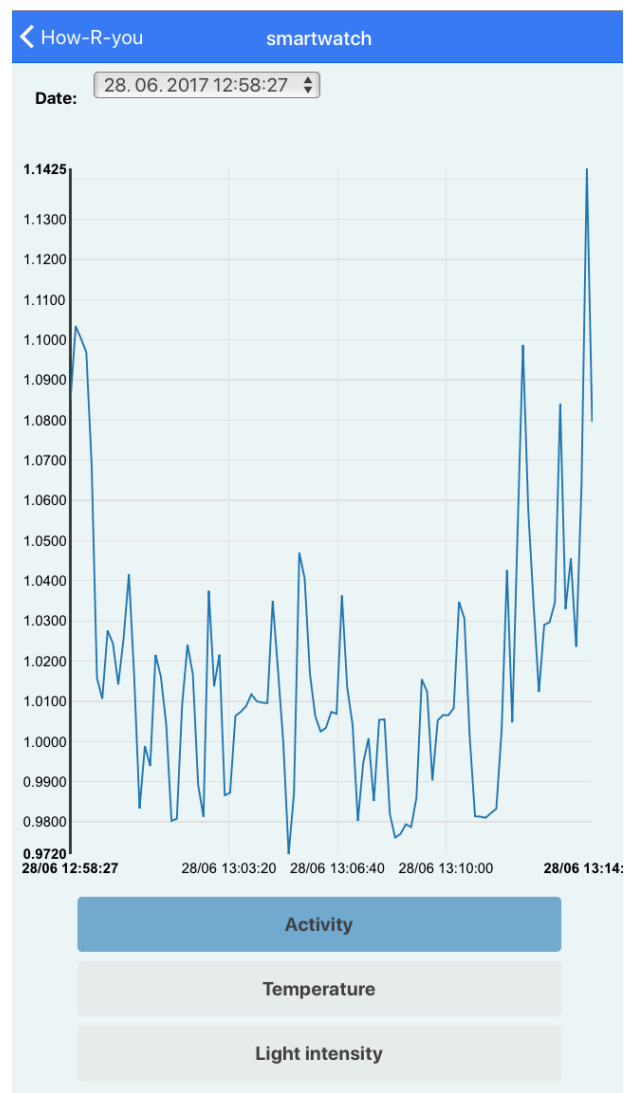

Figure 2. GENEActiv activity graph

The "Pain" module (see Figure 3) contains questions about pain intensity, where it is located, as well as the patient's level of physical activity, mood, and ability to participate in everyday activities. "Pain relief" focuses on methods and effects of relieving the pain, e.g. what the patient has done to alleviate the pain and what the outcome has been. The response format is an 0-10 slider scale (also known as Visual Analog Scale). The "Pain" questions are documented in the appendix.

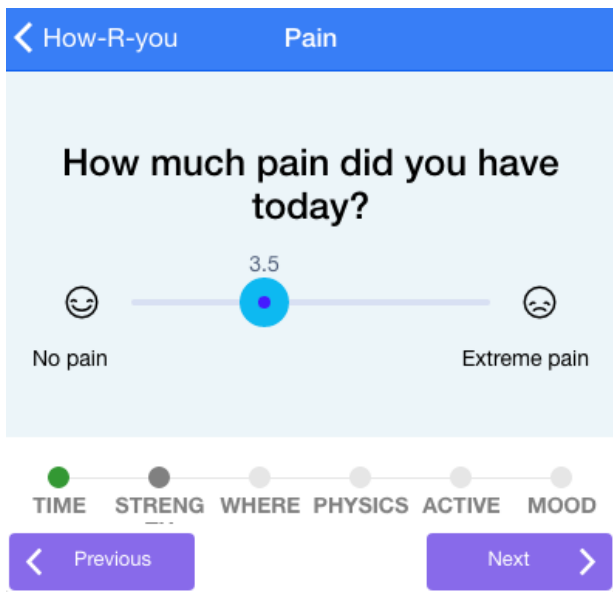

Figure 3. How-R-you "Pain" module

\subsection{Data collection}

As mentioned in the introduction, this paper is based on a research project that investigates the relationships between pain, sleep, and disease activity in an attempt to identify causes of reduced physical activities and pain among children and adolescents diagnosed with arthritis. The long-term goal is to identify effective methods of treatment and pain relief and as a consequence improve the quality of life among patients. 98 patients are included in the study "Pain, sleep and disturbed circadian rhythm in Juvenile Idiopathic Arthritis". It was determined that 85 would be sufficient to cover patients aged 6-16 years diagnosed with all 7 types of arthritis. The study has been approved by the regional committee on health research ethics and complies with applicable data protection laws. Informed consent was obtained from all participants and their guardians. The patients received instructions by a medical student and researcher.

Each patient participates over a three-month period, it includes two outpatient check-ups - one at inclusion and one by the end of the study - the purpose of which is routine assessments of disease activity. The patients were required to wear the GENEActiv device for the purpose of monitoring their level of physical activity and sleep pattern. They were also asked to write sleep diaries, fill out JAMAR questionnaires, and use the How-R-you app for one week following each of the two check-ups.

JAMAR is a paper-based questionnaire. In this study, the validated Danish translation of JAMAR was imported into REDCap, which is "a web application for building and managing online surveys and databases" (https://www.project-redcap.org). It was subsequently e-mailed to the parents of children included in the study. 
They were asked to fill out the JAMAR questionnaire on the day of the check-up. Furthermore, the patients were asked to download How-R-you, were provided with unique login information, and were asked to use the app daily. They were instructed to answer the app questions at least once a day for a week while wearing the GENEActiv device. All patients participated twice during the study - one week at the beginning of the research project and one week toward the end. The GENEActiv device served to identify each patient's circadian rhythm and ascertain whether this rhythm was affected by the patient's experienced pain. In evaluating and choosing between many different actigraphy devices, we opted for many sensory registrations per minute over a one-week period rather than fewer registrations over a longer period of time. Data from both weeks are included in the dataset. Each patient was assigned a unique ID in REDCap and stored along with How-R-you credentials.

All app activities and patient registrations (both in JAMAR and How-R-you) were recorded and timestamped. All data have been exported from the database for the purpose of data analysis using the Stata statistics software package (https://www.stata.com).

3.2.1. Ethics. In relation to our data collection, we discussed at length the potential risk and ethical concern of sickening the patients. By asking patients to daily monitor their health and respond to questions in the app, there is a possibility of constantly reminding the patients that they suffer from a chronic disease beyond the disease's normal cognitive saliency, which might influence their mood and mental well-being - and thus their state of health - in a negative way. For most patients the disease is, however, already a conscious part of their everyday lives. Furthermore, there is no wellestablished pain assessment method. Previous studies have confronted patients with variable number of questions about when patients experience pain [2]. For the purpose of this study we decided to monitor patients' health continuously and ask them to answer questions at least once a day. We therefore assess the risk as very low to the point where it is a concern that needs to be monitored as our ongoing research efforts move forward.

\subsection{Data analysis}

The analyses are based on cross sectional time-series data (panel data) since the data has been collected over two different time periods through, on the one hand, the JAMAR questionnaire and, on the other hand, the HowR-you app (December 2016 - November 2017).
Because of the nature of the data (cross sectional time-series data), the analyses are based on panel data models describing individual behavior over time and across individuals. There are several estimation techniques for analyzing panel data models, but at a general level there is a distinction between pooled Ordinary Least Squares (OLS) models, fixed effects models, and random effects models. We rely on both fixed and random effects models depending on the particular regression model, since Hausman specification tests have shown them to be the most appropriate and effective estimation techniques (for more about fixed and random effects, see [5]).

A Hausman specification test has been conducted for each regression model to determine if the specific regression model should be estimated using either fixed or random effects. Fixed effects are used if the p-value of the Hausman test falls below 0.05 (significance level), and random effects are used if the p-value exceeds 0.05 [1]. All analyses are based on the recognized 0.05 significance level $(p<0.05)$, but since the number of respondents are relatively low, relations at an 0.1 significance level $(\mathrm{p}<0.1)$ are highlighted as well. When $\mathrm{p}<0,001$, it is also emphasized. Each regression model displays the bivariate relationship between the study's independent (pain intensity) and dependent variables (level of physical activity, mood, and participation in activities) and is analyzed using Stata.

In the appendix, we have listed the questions that are compared across JAMAR and How-R-you. Despite differences in wording, the same questions (variables) are included in both the questionnaire and the app.

\section{Analysis}

In this section, we compare questionnaire (JAMAR) and app (How-R-you) responses through descriptive statistics and regression analysis. In the end, we conclude that the app as a PRO instrument provides for a more nuanced picture of patients' health.

If we compare JAMAR and How-R-you responses, we see some interesting results. In terms of descriptive statistics, $61 \%$ of all respondents have answered both JAMAR and How-R-you questions. 93 respondents have filled out the questionnaire and 66 have used the app.

The average registered pain intensity is higher when looking across questionnaire responses compared to the app data. However, the maximum registered pain is higher in the app than in the questionnaire. This is consistent with extant research, which shows that patients respond to questions regarding pain based on the maximum pain intensity experienced. 
Table 1 displays a comparison of patients' responses to questions regarding average pain intensity, level of physical activity, mood, and participation in activities in both the JAMAR questionnaire and the How-R-you app.

Table 1. Comparison of questionnaire and app responses

\begin{tabular}{lllll}
\hline & $\begin{array}{l}\text { Model I } \\
\text { Pain } \\
\text { intensity }\end{array}$ & $\begin{array}{l}\text { Model II } \\
\text { Physical activity }\end{array}$ & $\begin{array}{l}\text { Model } \\
\text { III } \\
\text { Mood }\end{array}$ & $\begin{array}{l}\text { Model } \\
\text { IIII } \\
\text { Activity }\end{array}$ \\
\hline App & $-0,588$ & $0,576^{*}(0,271)$ & $-0,633$ & $-1,582^{* *}$ \\
& $(0,422)$ & & $(0,386)$ & $(0,461)$ \\
Constant & $3,150^{* *}$ &,$- 060(0,432)$ & $2,273^{* *}$ & $\begin{array}{l}4,333^{* *} \\
(0,732)\end{array}$ \\
& $(0,672)$ & & $(0,613)$ & \\
\hline$n$ & 129 & 127 & 128 & 128 \\
$R^{2}$ & 0,015 & 0,035 & 0,021 & 0,086 \\
& & & &
\end{tabular}

$* p<0,05 ; * * p<0,001$. Reference category for the app is the questionnaire. The dependent variables are scaled from $0-10$, where 10 indicates a high impact of pain on level of physical activity, mood, and participation in activities, and conversely 0 indicates a low impact.

The respondents indicate that the experienced pain has influenced their level of physical activity (in terms of, e.g., running, jumping, writing, and cutting with scissors) - less so when asked in the questionnaire $(0,52$ on average) than in the app (1,09 on average). In other words, when asked daily about their health and wellbeing, the patients indicate being more impeded by pain than when asked to look back at the period since their last consultation. This might be a sign of a coping mechanism or simply that patients do not remember the pain influencing their level of activity.

When it comes to the experienced pain affecting their mood, the app score ( 1,01 on average) is lower than that of the questionnaire (1,64 on average). Meanwhile, the maximum registered value in the app (10) is identical to that of the questionnaire (10).

Similarly, looking at the extent to which the experienced pain has prevented the patients from participating in their usual activities (e.g., going to school, playing with friends, and participating in sports), the app score $(1,17$ on average) is lower than that of the questionnaire (2,75 on average). As with the impact of pain on their mood, the maximum registered value in the app (10) is identical to that of the questionnaire (10).

The regression analyses show that there is no significant difference between the registered pain intensity and mood across app and questionnaire. Looking at the beta coefficients, it can be inferred that the pain intensity and mood fall by $0.59(-0,59)$ and 0,63 $(-0,63)$ points respectively (NB: The dependent variable is scaled from 0 to 10) when transitioning from filling out the questionnaire to using the app. Opposite results are seen with regard to the level of physical activity and participation in activities in the sense that there are significant differences across app and questionnaire. The beta coefficients show that the level of physical activity increases by 0.58 points and participation in activities falls by $1,58(-1,58)$ points respectively when going from the questionnaire to the app. The results with regard to pain intensity, mood, and participation in activities are consistent with expectations of lower values registered in the app compared to the questionnaire.

Table 2 displays how the patients' pain intensity affects their level of physical activity, mood, and participation in activities.

Table 2. Effect of pain intensity on level of physical activity, mood, and participation in activities

\begin{tabular}{llll}
\hline & $\begin{array}{l}\text { Model I } \\
\text { Physical } \\
\text { activity (RE) }\end{array}$ & $\begin{array}{l}\text { Model II } \\
\text { Mood (RE) }\end{array}$ & $\begin{array}{l}\text { Model III } \\
\text { Activity } \\
\text { (RE) }\end{array}$ \\
\hline Pain intensity & $\begin{array}{l}0,303^{* *} \\
(0,054)\end{array}$ & $\begin{array}{l}0,435^{* *} \\
(0,074)\end{array}$ & $\begin{array}{l}0,631^{* *} \\
(0,088)\end{array}$ \\
Constant & $0,130(0,187)$ & 0,328 & $0,503 \dagger$ \\
& & $(0,248)$ & $(0,297)$ \\
\hline$n$ & 127 & 128 & 128 \\
Rho & 0,357 & 0,136 & 0,196 \\
\end{tabular}

$\dagger \mathrm{p}<0,1 ; * * \mathrm{p}<0,001$. Reference category for the app is the questionnaire. The dependent variables are scaled from $0-10$, where 10 indicates a high impact of pain on level of physical activity, mood, and participation in activities, and conversely 0 indicates a low impact.

The regression analyses show significant relationships between pain intensity, on the one side, and physical activity, mood, and participation in activities on the other side. The beta coefficient indicates that the impact of pain on level of physical activity rises 0,30 when the pain increases 1 point in intensity. For mood and participation in activities, the effects are 0,43 and 0,63 , respectively.

\section{Discussion}

This paper is the first step in addressing the need for more research that investigates the clinical value of PRO instruments (Lee et al. 2015) for the purpose of improving accuracy in patient data collection. In this paper, we look at the value from the perspective of doctors in evaluating whether the How-R-you app provides them with more valid and reliable information about patients' health compared to the periodic survey instrument JAMAR. Such information has the potential to improve medical work practices in support of diagnosis and treatment by enabling the doctors to make more informed and timely decisions based on more nuanced if not more accurate pictures of patients' health. 
The analyses show that even patients who are severely tormented by pain experience days with little or no pain. The app data allows for more detailed information about patients' health in the sense that the actual frequency of days with pain can be visualized together with the impact of pain on everyday life. As a consequence, doctors and patients have the means to recognize symptom patterns, indicating disease flare or the need to adjust pain treatment. Proper detection of a flare by comparing the associated pain with chronic pain is crucial for selecting the correct treatment. Early detection of a flare will signal a need for more intensive arthritis treatment. If the pain is chronic in nature, it is helpful to reflect on "good" as well as "bad" days, i.e. days with less versus more pain, because it may inform an effective treatment strategy. Daily app registrations of pain and how it affects patients in their daily lives provide doctors with a more nuanced picture of patients' health. In the future, this nuanced picture can be used to improve treatment and thereby the health of this patient group.

The well-known relationship between high pain intensity, low mood, decreased physical activity, and diminished social functioning [22] is captured by both JAMAR and How-R-you. However, some differences in patients' responses to questionnaire and app questions are significant, namely with regard to the level of physical activity and participation in activities, whereas others are not, specifically pain intensity and mood. This supports our assumption that patients answer JAMAR questions based on the most intensive pain they recall having experienced during the previous period. However, because the patients have used the app differently (some have used it several times a day and others have not used it every day despite the doctors' instructions), it is not possible to make any conclusions about pain variation over time across patients. Meanwhile, looking at individual patients' responses, for example with regard to pain intensity, substantial differences are clearly discernable, which again confirms our expectation that using the app supports a more nuanced picture of patients' health. This more nuanced picture of patients' health makes it possible to better evaluate whether certain pain management interventions (e.g., music therapy and meditation) have the desired effects. Moreover, with regard to the choice of treatment strategies (for example during "patientcentered talks"), the app data can be used because they allow for a discussion of day-to-day activities, and how they relate to experienced pain, medication, and much more.

Though we expected that the app facilitates a more accurate picture of patients' health, because they are asked to register data daily regarding their current health conditions compared to more retrospective evaluations over a longer period of time when they respond to the questionnaire, our analyses are inconclusive due to the fact that some of the identified differences are significant while others are not. However, there are clear advantages of using the app for collecting PRO data, not least the ability to establish a link between pain experienced now or very recently, patients' daily activities, and their well-being. In JAMAR, the patients answer many questions regarding their physical conditions that are not necessarily relevant to every child or adolescent. Moreover, because the answers relate to past experiences-sometimes weeks in the past - it is much more difficult to assess the impact of pain.

Although some differences across How-R-you and JAMAR are not significant, it is important to note the relatively small sample size, which may account for the non-significant differences in questionnaire and app responses. Looking at the individual responses, there are substantial differences in each patient's answers across JAMAR and How-R-you which confirms our expectation that the app facilitates a more nuanced-if not more accurate - picture of patients' health, and how pain influences their daily lives.

In summary, our study shows that digital PRO instruments like How-R-you can provide doctors with valid and reliable information in support of planning, monitoring, and improving treatment. In addition, such instruments may potentially increase patient satisfaction and compliance through their active involvement. Moreover, the PRO data may improve patient-doctor communication for the purpose of ensuring effective treatment strategies. However, since this study adopts the perspective of doctors, future studies are needed to investigate these potential values.

\subsection{Limitations}

In this study, we have investigated the potential value of using an app for collecting PRO data from the perspective of doctors. The data collected through the app helps doctors better diagnose diseases and monitor patients' health. In the end, because data is not collected automatically but needs to be registered manually by patients, it is important that patients are motivated, and that the app facilitates easy data registration. We are currently investigating the value perspective of patients. One of the envisioned benefits of using How-R-you is that patients have the possibility of monitoring their own medical conditions and reflecting on questions regarding their health (How much pain am I experiencing right now? How does it impact my physical condition and mental well-being?) in the hope that it may lead to pattern recognition (When did I experience it the last time? How did I cope? What was 
the effect?) and an improved ability to self-manage their disease on a daily basis. An important limitation of our study is that we do not control for the use of different technologies for data collection purposes. It is possible that the use of digital technologies (e.g., How-R-you) might influence not only data reporting but also the reported data and therefore the subsequent analysis. Future studies should consider including control variables such as computer self-efficacy, level of experience with digital technology, overall technology affinity etc.

Meanwhile, anecdotal evidence indicates that patients primarily use the app, because they want to help the doctors. Consequently, we need to better understand what motivates patients, and how they can be encouraged to take greater part in managing their own health, for example through technology use. To that end, we are studying the information needs of patients, and how digital technology like the app contributes to value creation from their perspective in order better to understand how to design and use such technologies. Our findings will be reported in forthcoming publications.

\section{Conclusion}

This paper reports on a study in which we set out to investigate the extent to which digital technology can be used to establish a more accurate picture of patients' health. We have collected PRO data using both a questionnaire and an app in a project that focuses on studying the relationships between pain, sleep, and disease activity among children and adolescents diagnosed with arthritis. We conclude that the use of digital technology (i.e., the app) allows for a more nuanced picture of patients' health to be established, and we discuss how the more detailed information can be used by doctors to better care for the patients through more effective treatment strategies and evaluation. In addition, we speculate that patients are better equipped to self-manage their disease. These advantages of using mobile digital technology have the potential to improve the health and quality of life of the patients. We will leave it to future studies to investigate this further.

\section{References}

[1] Baltagi, B., Econometric Analysis of Panel Data, John Wiley \& Sons Ltd, Chichester, England, 2013.

[2] Castarlenas, E., M. Jensen, C. von Baeyer, and J. Miró, "Psychometric Properties of the Numerical Rating Scale to Assess Self-Reported Pain Intensity in Children and
Adolescents: A Systematic Review", The Clinical Journal of Pain 33(4), 2017, pp. 376-383.

[3] Filocamo, G., A. Consolaro, B. Schiappapietra, et al., "A New Approach to Clinical Care of Juvenile Idiopathic Arthritis: The Juvenile Arthritis Multidimensional Assessment Report", The Journal of Rheumatology 38(5), 2011, pp. 938-953.

[4] Gallagher, J., J. O’Donoghue, and J. Car, "Managing immune diseases in the smartphone era: how have apps impacted disease management and their future?", Expert Review of Clinical Immunology 11(4), 2015, pp. 431-433.

[5] Gujarati, D., and D. Porter, Basic Econometrics, McGraw Hill Education, New York, NY, 2008.

[6] Hans, P., C. Gray, A. Gill, and J. Tiessen, "The provider perspective: investigating the effect of the Electronic PatientReported Outcome (ePRO) mobile application and portal on primary care provider workflow", Primary Health Care Research \& Development 19(2), 2018, pp. 151-164.

[7] Hays, R., "Healthcare apps", Australian Family Physician 45(11), 2016, pp. 849-850.

[8] Holmes, M., G. Lewith, D. Newell, J. Field, and F. Bishop, "The impact of patient-reported outcome measures in clinical practice for pain: a systematic review", Quality of Life Research 26(2), 2017, pp. 245-257.

[9] Iyawaa, G., M. Herselmana, and A. Botha, "Digital health innovation ecosystems: From systematic literature review to conceptual framework", Procedia Computer Science 100, 2016, pp. 244-252.

[10] Jepsen, T., "Doctor's Orders: Healthcare Apps and SelfMonitoring", IT Professional 16(4), 2014, pp. 48-49.

[11] Lee, Y., S. Shin, J. Kim, et al., "Evaluation of Mobile Health Applications Developed by a Tertiary Hospital as a Tool for Quality Improvement Breakthrough", Healthcare Informatics Research 21(4), 2015, pp. 299-306.

[12] Lomholt, J., M. Thastum, and T. Herlin, "Pain experience in children with juvenile idiopathic arthritis treated with anti-TNF agents compared to non-biologic standard treatment", Pediatric Rheumatology 11(21), 2013, pp. 1-8.

[13] LoPresti, M., M. Abraham, G. Appelboom, et al., "Clinical Trials Using Mobile Health Applications", Pharmaceutical Medicine 29(1), 2015, pp. 17-25.

[14] Martínez-Pérez, B., I. de la Torre-Díez, M. LópezCoronado, B. Sainz-de-Abajo, M. Robles, and J. GarcíaGómez, "Mobile Clinical Decision Support Systems and 
Applications: A Literature and Commercial Review", Journal of Medical Systems 38(4), 2014, pp. 1-10.

[15] McGowan, J., C. Cusack, and M. Bloomrosen, "The future of health IT innovation and informatics: a report from AMIA's 2010 policy meeting", Journal of the American Medical Informatics Association 19(3), 2012, pp. 460-467.

[16] McGrath, P., G. Walco, D. Turk, et al., "Core Outcome Domains and Measures for Pediatric Acute and Chronic/Recurrent Pain Clinical Trials: PedIMMPACT Recommendations", The Journal of Pain 9(9), 2008, pp. 771-783.

[17] Motamarri, S., S. Akter, P. Ray, and C. Tseng, 'Distinguishing 'mHealth' from Other Healthcare Services in a Developing Country: A Study from the Service Quality Perspective", Communications of the Association for Information Systems 34(Article 34), 2014, pp. 669-692.

[18] Nørgaard, M., M. Twilt, L. Andersen, and T. Herlin, "Accelerometry-based monitoring of daily physical activity in children with juvenile idiopathic arthritis", Scandinavian Journal of Rheumatology 45(3), 2016, pp. 179-87.

[19] Ramachandran, A., and V. Pai, "Patient-Centered Mobile Apps for Chronic Disease Management", International Conference on Computing for Sustainable Global Development (INDIACom), (2014), 948-952.

[20] Raphael, K., "Recall Bias: A Proposal for Assessment and Control", International Journal of Epidemiology 16(2), 1987, pp. 167-170.

\section{Appendix}

Table A1 and A2 contain the questions for PRO data collection across JAMAR and How-R-you.

\section{Table A1. JAMAR questions}

\footnotetext{
1 "How much pain have you had because of the illness over the past week?" (VAS 0-10; score of $0=$ "No pain" and score of 10 = "Extreme pain")

2 Evaluation of functional ability based on a JAFS (Juvenile Arthritis Functionality Scale) score; a 15-item questionnaire evaluating the child's ability to perform specific tasks during the past four weeks (each item is scored 0-2 where $0=$ "With NO difficulty", 1 = "With SOME difficulty", and 2 = "With MUCH difficulty"; total score range is $0-30$ )

3 "Consider the past four weeks, have you had any difficulty carrying out activities that require a lot of energy?" (4-point Likert-type scale; $0=$ "Never" and 3 = "Every day")

4 "Consider the past four weeks, have you felt sad or depressed?" (4-point Likert-type scale; $0=$ "Never" and $3=$ "Every day")
}

[21] Reiermann, J., "Professor: Potentialet ved patientinddragelse er grænseløst”, Mandag Morgen, September $11^{\text {th }} 2016$.

[22] Tarakci, E., I. Yeldan, E. Mutlu, S. Baydogan, and O. Kasapcopur, "The relationship between physical activity level, anxiety, depression, and functional ability in children and adolescents with juvenile idiopathic arthritis", Clinical Rheumatology 30(11), 2011, pp. 1415-1420.

[23] The Danish Ministry of Health, Local Government Denmark, and Danish Regions, Nationale mål for sundhedsvaesenet, 2017.

[24] The Economist, "Things are looking app", The Economist, March 10 2016.

[25] Ventola, C., "Mobile Devices and Apps for Health Care Professionals: Uses and Benefits", Pharmacy and Therapeutics 39(5), 2014, pp. 356-364.

[26] Wilson, L., "The World of Apps in Health Care", AJN, American Journal of Nursing 115(11), 2015, pp. 18-19.

[27] World Health Organization, Adherence to Long-Term Therapies: Evidence for Action, 2003.

[28] World Health Organization, mHealth: New horizons for health through mobile technologies: second global survey on eHealth, 2011.

\section{Table A2. How-R- you ("Pain") questions}

1 "Time" (time of day that the registration concerns is automatically detected but can be changed manually by the patient)

2 "How much pain did you experience today?" (Visual Analog Scale (VAS) $0-10$; score of $0=$ "No pain" and score of $10=$ "Extreme pain")

3 "Where does it hurt today?" (multiple choices: head, neck, back, legs, feet, arms, hands, and no pain)

4 "Does the pain mean you could not be as physically active as you would otherwise? (E.g., run, jump, write, cut with scissors)" (VAS $0-10 ; 0=$ "Not at all" and $10=$ "Completely prevented all activity")

$5 \quad$ "Has the pain prevented you from doing what you would otherwise do today? (E.g., school, play with comrades, go to sports)" (VAS 0-10; $0=$ "Not at all" and 10= "Completely prevented all activity")

6 "How much has the pain impacted your mood during the day?" (VAS 0-10; $0=$ "No impact" and 10= "Extreme impact") 